\title{
DERIVATION OF THE PROBABILITY DENSITY FUNCTION FOR ULTIMATE MUSCLE PH IN SLAUGHTERED ANIMALS
}

\author{
A. B. PLEASANTS ${ }^{1}$, G. C. WAKE ${ }^{2}$ and C. C. DALY ${ }^{1}$
}

(Received 12 February, 1999)

\begin{abstract}
We consider the form of the probability density function for the ultimate muscle $\mathrm{pH}$ in slaughtered animals. Muscle $\mathrm{pH}$ in slaughtered animals is dependent on a biochemical process which forms lactic acid from the breakdown of glycogen stored in the muscle at slaughter. The relationship between glycogen and muscle $\mathrm{pH}$ after slaughter is expressed as a pair of coupled differential equations. The solution of this system for the equilibrium muscle $\mathrm{pH}$ as time $\rightarrow \infty$ gives the form of the probability density for the ultimate muscle $\mathrm{pH}$. When the initial density for the muscle glycogen is normal, the density for the ultimate muscle $\mathrm{pH}$ is shown to be approximately a mixed normal density.
\end{abstract}

\section{Introduction}

During the conversion of muscle to meat post mortem, the dormant biochemical event is the gradual fall in muscle $\mathrm{pH}$, from approximately 7.2 in the living animal to, typically, 5.5. This gradual acidification of the muscle is the product of the conversion of muscle glycogen to lactic acid, a reaction that regenerates muscle adenosine triphosphate (ATP). Over a post mortem period of up to 12 hours in beef muscle, the drop in $\mathrm{pH}$ inhibits the resynthesis of ATP, and ATP concentrates decline; rigor mortis is defined as complete depletion of ATP (see Bendall [2] for a review of the post mortem changes in muscle), and the $\mathrm{pH}$ of meat at rigor mortis as the ultimate $\mathrm{pH}\left(\mathrm{pH}_{u}\right)$.

The $\mathrm{pH}_{u}$ of meat has important commercial consequences. Compared with meat with a normal $\mathrm{pH}_{u}$ of 5.5 , an increase in the $\mathrm{pH}_{u}$ of meat adversely affects colour, flavour, texture and shelf life ([6]). There are therefore strong commercial justifications

\footnotetext{
${ }^{1}$ AgResearch, Private Bag 3123, Hamilton, New Zealand; e-mail: tony.pleasants@agresearch.co.nz, clyde.daly@agresearch.co.nz.

${ }^{2}$ Department of Mathematics and Statistics, The University of Canterbury, Private Bag 4800,

Christchurch, New Zealand; e-mail: graeme.wake@canterbury.ac.nz.

(C) Australian Mathematical Society 2003, Serial-fee code 1446-1811/03
} 


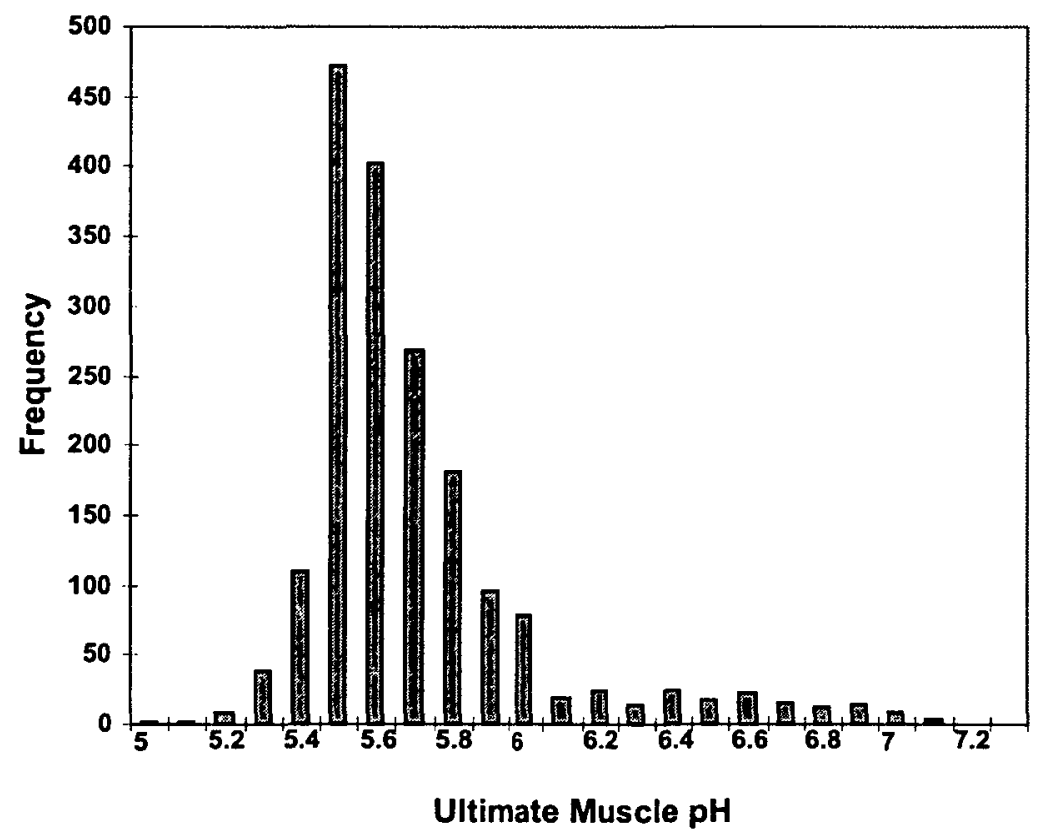

FIGURE 1. Ultimate muscle pH for steers slaughtered at Manawatu Beef Packers during 1993/94.

for understanding and controlling the causes of elevated $\mathrm{pH}_{u}$ in meat. The $\mathrm{pH}_{u}$ is related to the concentration of glycogen in muscle at slaughter. Approximately $40 \mu \mathrm{moV} / \mathrm{g}$ of muscle tissue are needed to lower the $\mathrm{pH}$ from 7.2 to 5.5. If the concentration of glycogen at slaughter is higher than this value, the residual is not metabolised. When lower than this value, the $\mathrm{pH}$ decline is arrested by the lack of substrate (glycogen) and the $\mathrm{pH}_{u}$ is elevated. The relationship between $\mathrm{pH}_{u}$ and initial glycogen concentration is linear for values of initial glycogen less than $40 \mu \mathrm{mol} / \mathrm{g}$ of muscle, and the slope is proportional to the buffering capacity of the muscle tissue. Muscle glycogen concentrations at slaughter are reduced primarily by stress in the period before slaughter [6].

Efforts to understand the factors contributing to high $\mathrm{pH}_{u}$ in meat have been hampered by the nature of the frequency distribution of muscle $\mathrm{pH}_{u}$ in a population of slaughtered animals. Figure 1 shows a typical frequency distribution that exhibits a peaked primary mode with a long upper tail and a suggestion of a secondary mode at high $\mathrm{pH}_{u}$. While the definition of $\mathrm{pH}$ in terms of the hydrogen ion concentration involves logarithms, taking inverse logarithms or any other simple transformation of the $\mathrm{pH}_{u}$ data fails to derive a normal probability density.

Farmers would like to introduce strategies to breed animals with genetic potential for low ultimate muscle $\mathrm{pH}$. Also meat processors would like to identify farmers who 
can consistently supply animals with low $\mathrm{pH}_{u}$ for slaughter. Both these problems need good estimates of the repeatability of the $\mathrm{pH}_{u}$, to be successful. Good estimates of the repeatability require a good theoretical understanding of the probability density of the trait in question.

This paper derives a probability density for the ultimate muscle $\mathrm{pH}_{u}$ in slaughtered animals from some basic facts about the breakdown of muscle glycogen to lactic acid in the muscle after slaughter. Using this probability density, improved estimates of the repeatability of muscle $\mathrm{pH}_{u}$ between sires (heritability) and between suppliers can be made.

\section{Derivation of the probability density for ultimate muscle pH in slaughtered animals}

The biochemical pathway from muscle glycogen to lactic acid (which determines muscle $\mathrm{pH}_{u}$ ) involves a complex series of enzymatic reactions (Lehninger [4]). However, since the end product, lactic acid, is the only aspect of the pathway of interest, the primary dynamics can be described by how the input (muscle glycogen) and the output (lactic acid) change over time. These dynamics can be described by how the input (muscle glycogen) and the output (lactic acid) change over time. These dynamics can be approximated by:

- The muscle $\mathrm{pH}$ and the availability of muscle glycogen determine the rate of change of muscle glycogen over time. As the muscle $\mathrm{pH}$ falls the breakdown of muscle glycogen falls until, at $\mathrm{pH}=5.5$, no further muscle glycogen is broken down.

- The rate of change of $\mathrm{pH}$ in the muscle also depends on the availability of muscle glycogen and the buffering properties. Close to a muscle $\mathrm{pH}$ of 7.2 , buffering capacity is high so that a proportion of hydrogen ions generated by glycolysis are bound and $\mathrm{pH}$ falls slowly. Similarly as muscle $\mathrm{pH}$ reaches 5.5 glycolysis becomes increasingly inhibited by the $\mathrm{pH}$ so that the rate of $\mathrm{pH}$ change slows, finally ceasing at a $\mathrm{pH}$ of 5.5 .

These factors can be expressed mathematically in terms of the following pair of differential equations. Here $p$ designates muscle $\mathrm{pH}$ and $G$ designates muscle glycogen. The rate of change of both muscle $\mathrm{pH}$ and muscle glycogen depends on the same function of the amount of muscle glycogen $y(G)$ expressed as a mass ratio:

$$
\begin{aligned}
\frac{d G}{d t} & =(5.5-p) y(G), \\
k \frac{d p}{d t} & =(5.5-p)(7.2-p) y(G),
\end{aligned}
$$

where $k$ is a constant related to the rate of each equation. The units of $G$ are scaled so that $k=1$. 


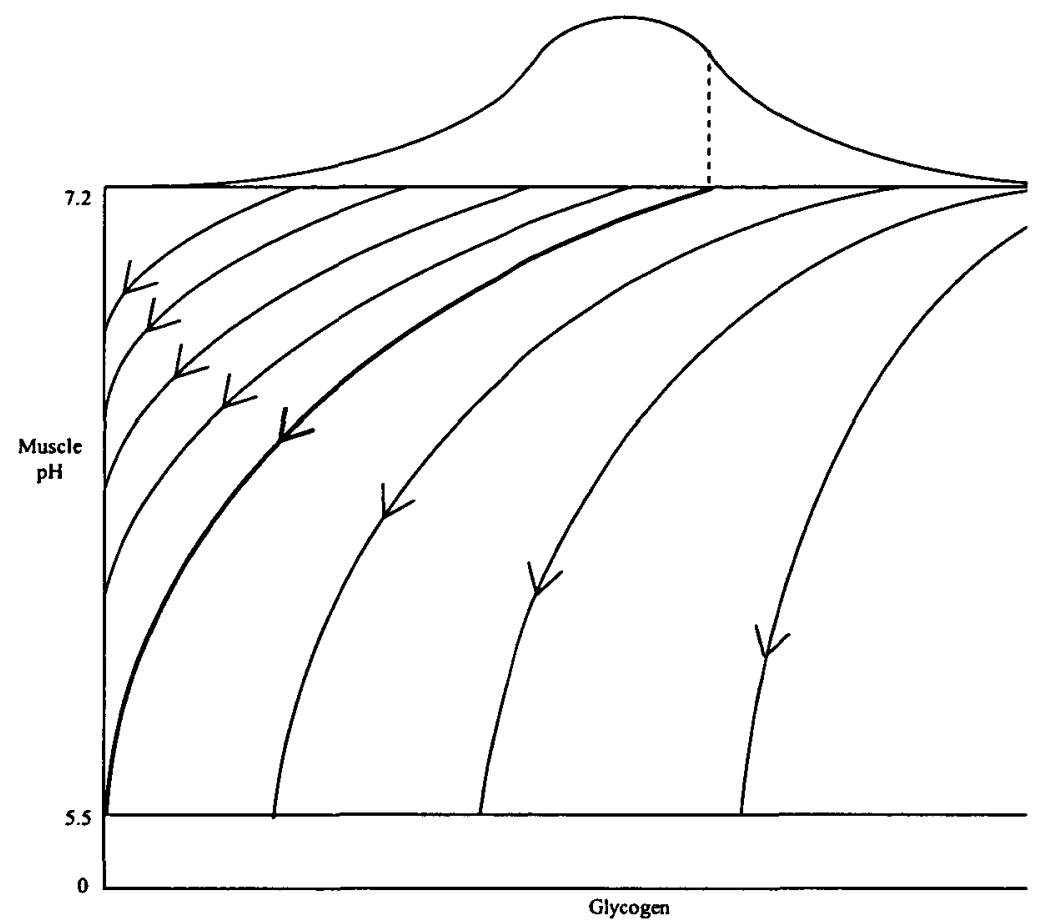

FIGURE 2. The phase plane for the system of differential equations (2.1) describing the evolution of muscle $\mathrm{pH}$ in meat after slaughter for a normal distribution of initial glycogen levels.

This system has a set of equilibrium points. Either $y(G)=0$ and $5.5 \leq p \leq 7.2$ or $p=5.5$ and $y(G)>0$. That is, a separate equilibrium point exists for every set of initial conditions. Depending on the initial amount of glycogen and the initial $\mathrm{pH}$ the equilibrium state of the $\mathrm{pH}$ in the muscle will either be at 5.5 , or at a $\mathrm{pH}$ of greater than 5.5 depending on the initial amount of glycogen available. The streamlines of the phase plane for this systems are shown in Figure 2.

From (2.1) the equation for the streamlines in the $\mathrm{pH}$ glycogen phase plane shown in Figure 2 is given by

$$
\frac{d p}{d G}=7.2-p
$$

This equation has the solution

$$
p=7.2-C e^{-G},
$$

where $C$ is related to the amount of glycogen in the muscle at slaughter, that is, at time $t=0$, and $p(t=0)=7.2-\epsilon$ where the constant $\epsilon$ allows for the fact that the muscle 
$\mathrm{pH} p$ differs from 7.2 in the live animal, arbitrarily close from below. By writing the initial condition into (2.2) we get

$$
p=7.2-\epsilon e^{G_{0}-G}
$$

This relates the value of $G_{0}$ at slaughter to the initial $\mathrm{pH}$ value of 7.2.

To achieve a ultimate muscle $\mathrm{pH}$ of $5.5,(2.2)$ shows that for $G \geq 0$,

$$
G_{0} \geq \ln (1.7 / \epsilon)=Q
$$

Let the probability density of muscle glycogen in a population of live animals be $f\left(G_{0}\right)$ at time $t=0$. Then from the derivation above,

$$
\operatorname{Prob}[\mathrm{pH}=5.5]=\int_{Q}^{\infty} f\left(G_{0}\right) d G_{0} .
$$

Thus a probability density for the ultimate muscle $\mathrm{pH}$ in slaughtered animals will be

$$
P[p]=\left\{\begin{array}{l}
\int_{Q}^{\infty} f\left(G_{0}\right) d G_{0} \\
\beta \varepsilon e^{G_{0}} f\left(G_{0}\right)
\end{array}\right.
$$

where $\beta$ is chosen to make $P(p)$ a probability density function and $G_{0}$ depends on $p$. This follows since if $p=F\left(G_{0}\right)$ and $G_{0}$ has a probability density function of $f\left(G_{0}\right)$ then $p$ has a probability density function proportional to $F^{\prime}\left(G_{0}\right) \cdot f\left(G_{0}\right)$.

The measurement of muscle $\mathrm{pH}$ in the slaughtered animal involves instrument error. Assume that this instrument error is a normally distributed random variable with mean zero and variance $\sigma_{m}^{2}$. Then the observed probability density for the ultimate muscle $\mathrm{pH}$ in a slaughtered animal will be the convolution of the instrument error density with the density for the actual ultimate muscle $\mathrm{pH}$ :

$$
P[p]=N\left(5.5, \sigma_{m}^{2}\right) \int_{Q}^{\infty} f\left(G_{0}\right) d G_{0}+\left.\left(\beta \varepsilon e^{G_{0}} f\left(G_{0}\right) * N\left(0, \sigma_{m}^{2}\right)\right)\right|_{G_{0}=0} ^{Q},
$$

where $*$ is the convolution operator, and the last term indicates that $G_{0}$ is truncated at $Q$.

Let $\alpha=\int_{q}^{\infty} f\left(G_{0}\right) d G_{0}$. Then

$$
P[p]=\alpha N\left(5.5, \sigma_{m}^{2}\right)+\left.(1-\alpha) \gamma e^{G_{0}} f\left(G_{0}\right) * N\left(0, \sigma_{m}^{2}\right)\right|_{G_{0}=0} ^{Q} .
$$

If the $\mathrm{pH}$ buffers of 5.5 above and 7.2 below are taken as normally distributed random variables, then the steady states reached by different animals will vary for the same initial amount of glycogen. This variability is factored into the construction of the probability density (2.4) before the truncation into the mixed density takes place. If 
an approximation is made by including measurement error as part of the variation associated with the buffer uncertainty then the probability density can be expressed as a mixed normal density. Assume that the probability density of muscle glycogen in the live animal is normal, then making the transformation from $p$ to $G_{0}$ by $G_{0}=$ $\ln (7.2-p)$, we obtain

$$
P[p]=\alpha N\left(\ln (1.7), \sigma_{m}^{2}\right)+(1-\alpha) N\left(\mu_{\mathrm{ln}}, \sigma_{m}^{2}+\sigma_{\mathrm{lp}}^{2}\right),
$$

where $\mu_{\mathrm{n}}$ is the mean of $\ln (7.2-p)$ and $\sigma_{\mathrm{lp}}^{2}$ is the variance of $\ln (7.2-p)$.

Hosmer [3] has considered the theoretical issues in obtaining maximum likelihood estimates of the parameters of the mixed normal density and gives an iterative algorithm for the solution. Agha and Ibrahim [1] present a computer program in Algol for these estimates.

The parameters to be estimated are

- $\mu$ : The expected value of the amount of muscle glycogen in the population of animals before slaughter;

- $\sigma_{x}^{2}$ : The variance of the amount of muscle glycogen in the population of animals before slaughter; and

- $\sigma_{m}^{2}$ : The variance of the measurement of the ultimate muscle $\mathrm{pH}$ in the slaughtered animals.

The parameter $\alpha$ is obtained by letting $Q=\ln (1.7)$.

\section{Application to measuring repeatability between suppliers}

Since the ultimate muscle $\mathrm{pH}$ in the slaughtered animal is related to meat quality it is of interest to a meat processor to be able to rank suppliers for this trait. An effective ranking system depends on a degree of repeatability within a supplier. Repeatability is defined as the intraclass correlation for the trait of interest.

The ultimate muscle $\mathrm{pH}$ in 1822 slaughtered animals supplied by 16 farmer suppliers to Manawatu Beef Packers, a meat processing company in New Zealand, was used to estimate the above parameters of the probability density for the ultimate muscle $\mathrm{pH}$ using maximum likelihood. The estimated parameters, using probability density (2.4), were

$$
\mu=1.31 \pm 0.002, \quad \sigma_{x}=0.041 \pm 0.001, \quad \sigma_{m}=0.316 \pm 0.006 .
$$

The standard errors of the parameters were obtained by a bootstrap.

Previously when the repeatability for the ultimate muscle $\mathrm{pH}$ within suppliers was calculated ignoring the form of the probability density the estimate was not signifcantly different from zero, suggesting that there was no effective ranking of suppliers. 
However, when the repeatability was calculated assuming a normal probability density for muscle glycogen in the animal before slaughter and estimating the parameters of this density using the above techniques the repeatability was calculated to be 0.47 . An effective method of ranking suppliers can now be designed.

It is suspected that animal husbandry practices may affect the level of glycogen in the animal before slaughter and therefore the ultimate muscle $\mathrm{pH}$ and the quality of the meat. However, investigations of various practices have not identified aspects which have an effect on the ultimate muscle $\mathrm{pH}$ in the slaughtered animal (Purchas and Keohane, [5]). Applying the probability density (2.4) or (2.5) to obtain maximum likelihood estimates of the effects of interest would give more power to these investigations.

\section{Discussion}

This paper has referred to a specific problem, the determination of the probability density of the observed equilibrium muscle $\mathrm{pH}$ in slaughtered animals. However, any system which is buffered in the manner described above and in which one component may become limiting would be expected to show similar equilibrium probability densities.

In retrospect it is clear how the technique works. Most of the variation around the peak of the density represents instrument error. That is, information on differences in muscle glycogen in these animals has been lost. Information on differences between animals is to be found primarily in the tail of the frequency distribution. In effect the maximum likelihood procedure derived from (2.4) discounts the information in this part of the distribution in favour of information in the tail of the distribution.

\section{Acknowledgements}

The authors are grateful to Dr Simon Woodward for helpful discussion and to Dr Neil Cox for assistance and discussion.

\section{References}

[1] M. Agha and M. T. Ibrahim, "Maximum likelihood estimation of mixtures of distributions. Algorithm AS 203", Appl. Stat. 32 (1984) 327-332.

[2] J. R. Bendall, "Post mortem changes in muscle", in The structure and function of muscle (ed. G. H. Bourne), Volume II, (Academic Press, New York, 1973) 244-306. 
[3] D. W. Hosmer, "A comparison of iterative maximum likelihood estimates of the parameters of a mixture of two normal distributions under three different types of sample", Biometrics 29 (1973) $761-770$.

[4] A. L. Lehninger, Principles of biochemistry (Worth Publ., New York, 1982).

[5] R. W. Purchas and B. E. Keohane, "Sources of variation in the ultimate muscle pH of M. Longissimus from prime steers", Proc. New Zealand Soc. Animal Production 57 (1997) 271-277.

[6] P. V. Tarrant and D. E. Hood, The problems of dark-cutting in beef (Marinus Nijhoff, Den Haag, 1981). 